\title{
On-line Inspection of the Surface Roughness of Workpiece in Ultraprecision Machining
}

\author{
Hsi-Hsun Tsai ${ }^{1}$ and Hui-Ping Feng ${ }^{2}$ \\ ${ }^{1}$ Associate Professor, ${ }^{2}$ Professor \\ Graduate Institute of Electro-Mechanical Engineering, Ming Chi University of Technology \\ 84 Gungjen Rd, 243 Taisun, Taipei County, Taiwan \\ ${ }^{1}$ hhtsai@mail.mcut.edu.tw, ${ }^{2}$ hpfeng@mail.mcut.edu.tw \\ Tel:886-229089899\#4515
}

Fax:886-229063269

Keywords: surface roughness, coherent light scattering, ultraprecision machining, photodiode

\begin{abstract}
An in situ technique to determine the surface roughness of ultraprecision machining using optical characteristic effects is fundamental as probes are not used, which prevents contact damage on the surface. Because the plastic lens molding reprints the roughness of the mold core fabricated by machining, tool marks result in the poor surface of the plastic lens. The machined surface can reflect the input light of a green laser with a short wavelength of $532 \mathrm{~nm}$. By varying the feed rate of the ultraprecision machining, several samples of the electrolyte-less nickel with different surface roughness have been examined using the green laser and the photodiode array. The distribution of the optical scattering effect of each sample under the light source of green laser is derived for prediction of the surface roughness. The results show that greater surface roughness produces more expansive distribution of light scattering. In addition, the bidirectional scatter distribution function (BSDF) of the machined surface is found to be proportional to roughness. Using the ratio of the main and the side measuring channels of the photodiode array, a suitable approach to establish the relationship between light scattering and surface roughness can be developed. The laser and the photodiode array are found to efficiently predict the surface roughness of the ultraprecision machined electroless nickel.
\end{abstract}

\section{Introduction}

Surface roughness is typically used to describe the level of the machining mark on the workpiece. Generally, a stylus measures the surface roughness of the machined workpiece in industrial applications. However, this contact method may damage the workpiece surface and does not help in the complete inspection of the workpieces. Hence, a noncontact method for measuring the surface roughness is vital. Among the noncontact methods, optics-based approaches, including by light scattering and digital image approaches, are the primary noncontact methods for surface roughness measurement.

The injection molding process reprints the surface roughness of machining from the mold core to the plastic lens. The reprinted surface roughness of the plastic lens produces light scattering effect, while the light is transmitted to and/or reflected from the interface of the plastic and air. Thus, the induced light scattering produces stray light in the optical system, which affects the performance of the lens system. Theoretical investigation of light scattering induced by surface roughness is complicated, due to the fluctuation in the profile with numerous irregularities. Zhao and Gao (2009) indicated that the speckle contrast method and the light scattering method are two of the most noncontact techniques for on-line surface roughness measurement of slightly-rough surface. The influence from the diffuse component of scattered light may eliminate. 
Many studies have been carried out to investigate light scattering induced by surface roughness, including those by Tanner and Fahoum (1976), who measured the reflected light intensity from the mirror surface to derive the relationship between roughness and scattering effect by the angular resolved method. The measured reflected light intensity from the mirror surface to derive the relationship between roughness and scattering effect. Beckmann (1987) constructed model of the ratio of the scattering intensity to the reflected light intensity by launching the laser on the surface while considering the roughness magnitude and the related characteristic surface parameters. Based on the Beckmann model, Persson (1998) used an in-process measuring scheme to predict the surface roughness in grinding with a laser. Vandembroucq et al. (2001) revealed that previous research incorrectly measured the surface roughness and the induced light scattering by neglecting the effects of multi-scattering and shadowing. The light scattering technique can be used to predict the surface roughness of steel in the turning process (Wang et al., 2000) and of a silicon wafer in the grinding process (Tay et al., 2004).

To derive the relationship between surface roughness and scattering effect, Fontani et al. [2000] measured the light scattering of the machining parts with flat surface by eight photodiode sensors and $\mathrm{He}-\mathrm{Ne}$ laser of $632.8 \mathrm{~nm}$ wavelength. These sensors recorded $90^{\circ}$ radial distribution of scattering light. Furthermore, Fontani et al. [2001] used a linear array of sensing detector with 16 photodiodes to derive the ratio of scattering to reflective light intensity induced by the machining mark on the crankshaft. The surface roughness of the crankshaft was predicted using this ratio. However, the positioning of the light source and the sensing detector was more complicated.

The mold core surface, subjected to ultraprecision machining, is usually assumed to be mirrorlike for the design of plastic light-guide components. However, the tool marks during machining can cause poor surface of the plastic lens. Beckmann [5] proposed the following model for the description of electromagnetic scattering induced by random scratching surface:

$\frac{I_{S}}{I_{O}} \propto\left[-\left(\frac{4 \pi R_{q} \cos \theta}{\lambda}\right)^{2}\right] \quad$ for $\frac{I_{S}}{I_{O}}>0.6$

where $I_{S}$ is the mirror specular light intensity, $I_{O}$ is the whole reflective light intensity, $\theta$ is the incident angle, and $R_{q}$ is the root mean square of surface roughness.

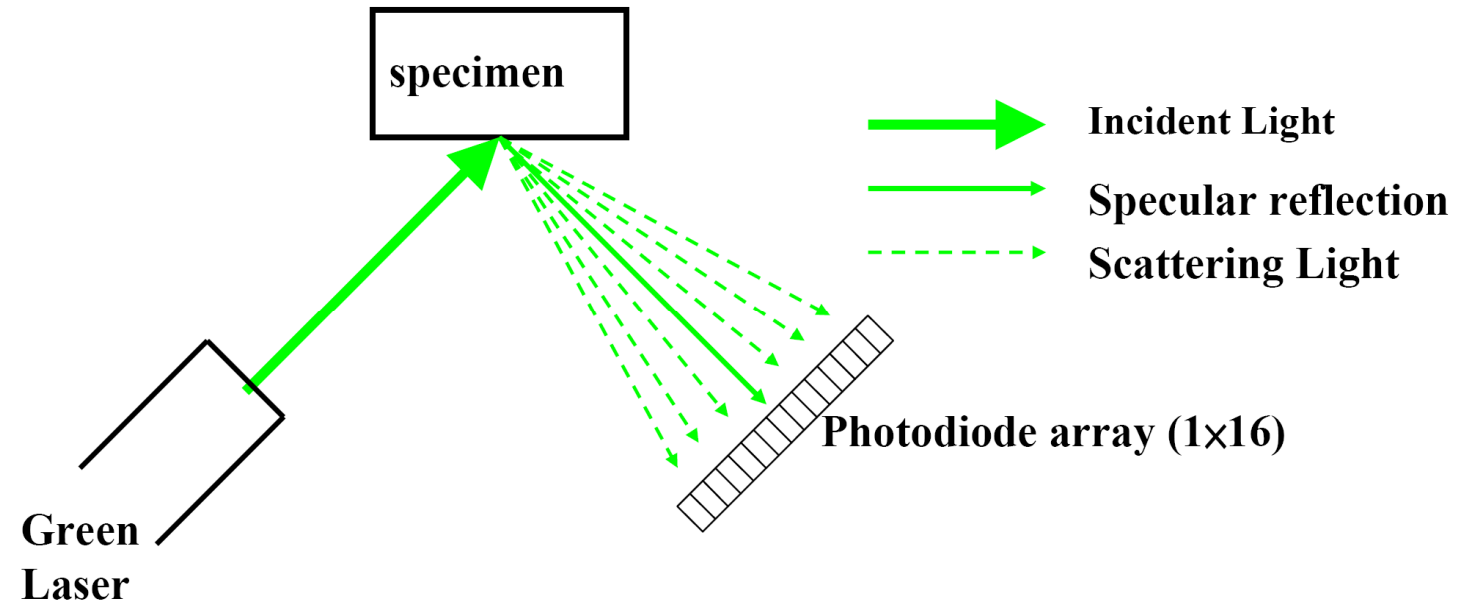

Fig. 1 Incident angle, light scattering angle and the reflective specular light

The specular light intensity from the surface is decreased, while the scattering light intensity is increased, with respect to the increased roughness. Using the ratio of scattering light intensity near 
the imposed point to the one far from the imposed point, Cahill [2001] tried to derive the roughness of the machining surface. As analysis of the light scattering effect induced by surface roughness is sophisticated, Church et al. [1977] first predicted the roughness by the bidirectional scatter distribution function $(B S D F)$. However, Stover [1995] showed that light scattering of the interface of media can be defined by either BSDF or total integrated scattering (TIS). The BSDF can be obtained using the following expression: $B S D F=$ differential radianceldifferential irradiance. In Fig. 1, the irradiance indicates the ratio of the laser power imposed on the spot of the surface to the area of the spot $\left(\boldsymbol{P}_{\text {laser }} / \boldsymbol{A}_{\text {spot }}\right)$, and thus, the radiance can be given as

radiance $=P_{\text {scatter }} /\left(\Omega_{s} \cos \theta_{s} A_{\text {det ector }}\right)$

where $\Omega_{s}$ is the solid angle, $P_{\text {scatter }}$ is the scattering light intensity of the photosensor received, $\theta_{s}$ is the scattering angle, and $A_{\text {detector }}$ is the receiving area of the photosensor. Hence,

$$
\begin{aligned}
B S D F & =\left(P_{\text {scatter }} / \Omega_{s} \cos \theta A_{\text {det ector }}\right) /\left(P_{\text {laser }} / A_{\text {spot }}\right) \\
& =P_{\text {scatter }} A_{\text {spot }} / \Omega_{s} \cos \theta A_{\text {detector }} P_{\text {laser }}
\end{aligned}
$$

Once $A_{\text {det ector }}=\boldsymbol{A}_{\text {spot }}$, Eq. (3) can be simplified as $B S D F=\left(P_{\text {scatter }} / P_{i} \Omega_{s} \cos \theta_{s}\right)$. In fact, for a metal workpiece, the main scattering occurs from the reflectance.

The BSDF can be simplified as BRDF (Bidirectional Reflectance Distribution Function), by neglecting the transmission into the interface. For ultraprecision machining process, the surface roughness of the workpiece is important during the application of plastic lens molding. As contact probing is used to determine the surface roughness, which may damage the mirror-like surface, a contactless measurement technique can thus be useful for the ultraprecision process. However, in the interferometer approach, the measurement cost is more expensive owing to the higher equipment cost. As scattering effect can help determine surface roughness, Beckmann [1987], Persson [1998], and Vandembroucq et al. [2001] tried to predict the surface roughness using laser scattering.

Elson and Bennett [1989] showed that if the irregularity size of the surface profile is similar to or smaller than that of the reflected light, then light scattering should be analyzed by refraction theory. They found that the angular distribution of scattered light from optical surfaces as a function of the wavelength. However, there is no literature on the surface roughness measurement for ultraprecision machining using light scattering effect of green laser. Shorter wavelength than the He-Ne laser, the green laser may give an accurate solution in this study. Hence, the purpose of this study was to investigate the surface roughness of ultraprecision workpiece using light scattering effect. Several samples of electroless nickel with different surface roughness obtained by varying the feed rate of the ultraprecision machining were examined by the green laser. The $1 \times 16$ photodiode array with a 2-mm pitch was constructed to measure the distribution of the optical scattering effect under the light source of the green laser.

\section{Experimental Setup}

In this study, electroless nickel workpieces were machined using an ultraprecision lathe to derive different levels of surface roughness. Fixing the the revolution frequency of the workpiece at $2400 \mathrm{rpm}$ and the cutting depth of the diamond tool at $1 \mu \mathrm{m}$, the different levels of surface roughness derived from varying the feedrate of the diamond tool. On pointing the coherent laser on the surface of the workpieces, the photodiode array was able to detect the light scattering effects of the samples with different surface roughnesses. The wavelength and power of coherent green laser are $532 \mathrm{~nm}$ and $5 \mathrm{~mW}$, respectively. Besides, the S5668 Hamamatsu silicon photodiode array of had a $1 \times 16$ linear of pitch of $1.6 \mathrm{~mm}$. With the coherent laser's incident angle on the surface of the 
workpieces, the reflected specular light from the surface was set on the 8th channel of the photodiode array to describe the scattering distribution. An Advantech PCI-1710 AD/DA acquisition card was used to transfer the sensing voltage of each element of the photodiode array to the desktop computer.

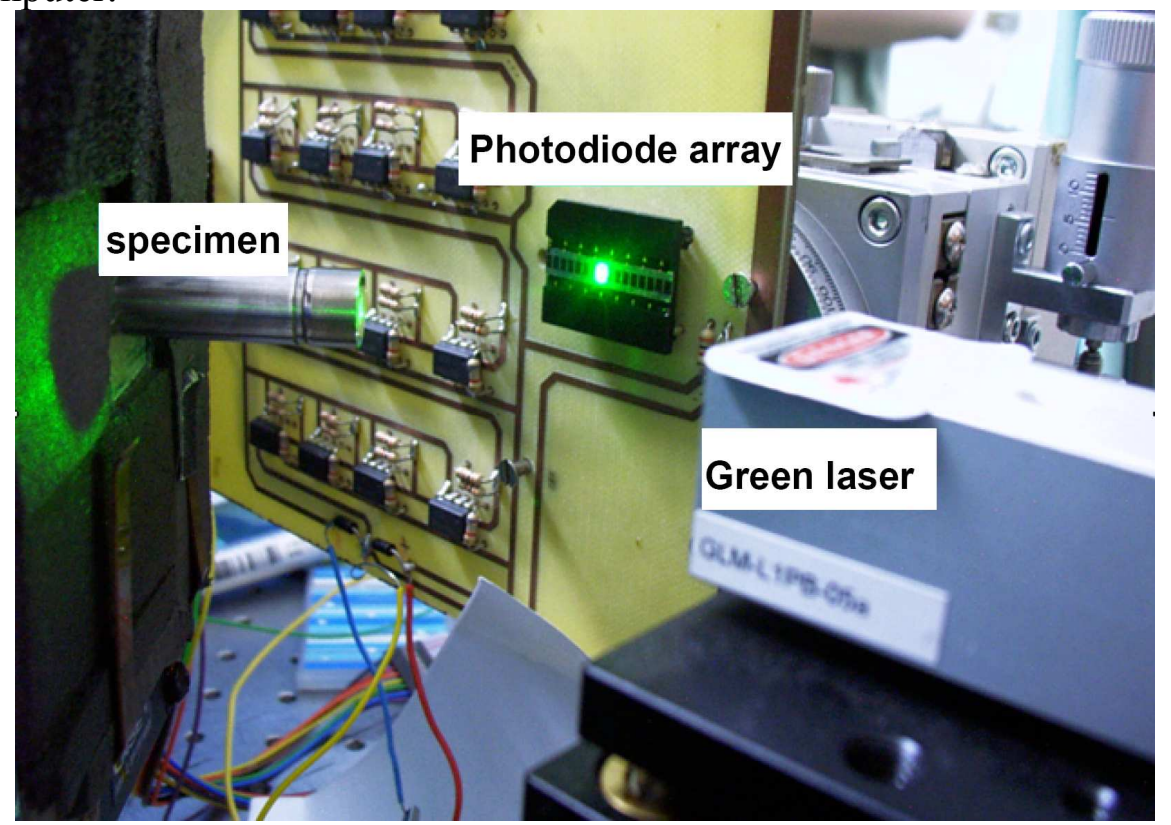

Fig. 2 The experimental setup includes the green laser, a workpiece, a photodiode array and amplified circuits

Figure 2 shows the experimental setup where the green laser has an incident angle of $45^{\circ}$ to the machined end surface of the electroless nickel workpiece. According to the Fresnel theorem, the incident angle equals the reflective angle where the normal line is perpendicular to the machined end surface of the workpiece. The reflected light with scattering effect was collected by the photodiode array. The distance between the workpiece and the photodiode was either 26 or $50 \mathrm{~mm}$. Figure 3 shows the scattering effect induced by surface roughness $(\mathrm{Rq})$ of $48.7 \mathrm{~nm}$ by green laser. The light has been spread expansively associated with the diffractive effect by the surface roughness of the workpiece in ultraprecision machining, which is in agreement with the previous literature findings. 


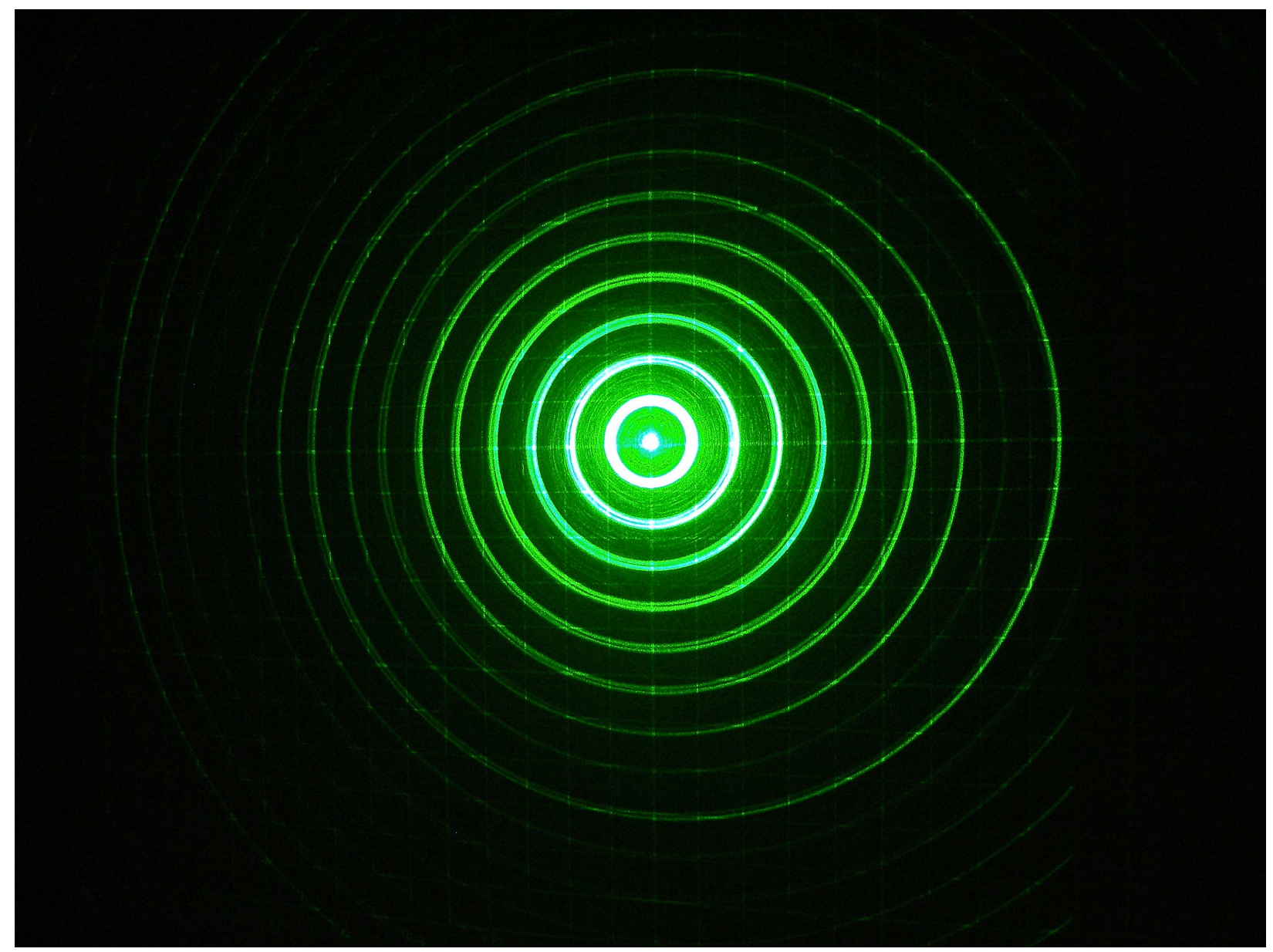

Fig. 3 The scattering effect induced by surface roughnesses (Rq) of $48.7 \mathrm{~nm}$ by green laser

\section{Results and Discussion}

In the previous experimental setup of laser incident angle of $45^{\circ}$ and different levels of the surface roughness workpiece, the photodiode array measured the scattering distribution from the end surface of the workpiece at distances of 26 and $50 \mathrm{~mm}$. Figure 4 shows that the received intensity (voltage) of the specular channel increased with the lower surface roughness. The regressive equation fits well to the row experimental data thanks to the higher $\mathrm{R}^{2}$. By the equation, the received intensity from the rough surface under ten percent of the wavelength of incident green laser is 3.679 (V) while the perfect surface without the roughness gives the received intensity is $4.215(\mathrm{~V})$. The analysis reveals that the surface roughness below one tenth of the incident light wavelength induces about $13 \%$ loss of the specular light. Due to the plastic injection molding is an imprint process, the surface roughness of the mould is printed on the molding parts. The surface roughness requirement of the plastic optical lens should be more restrict for the higher specular light intensity and the lower light scattering. 


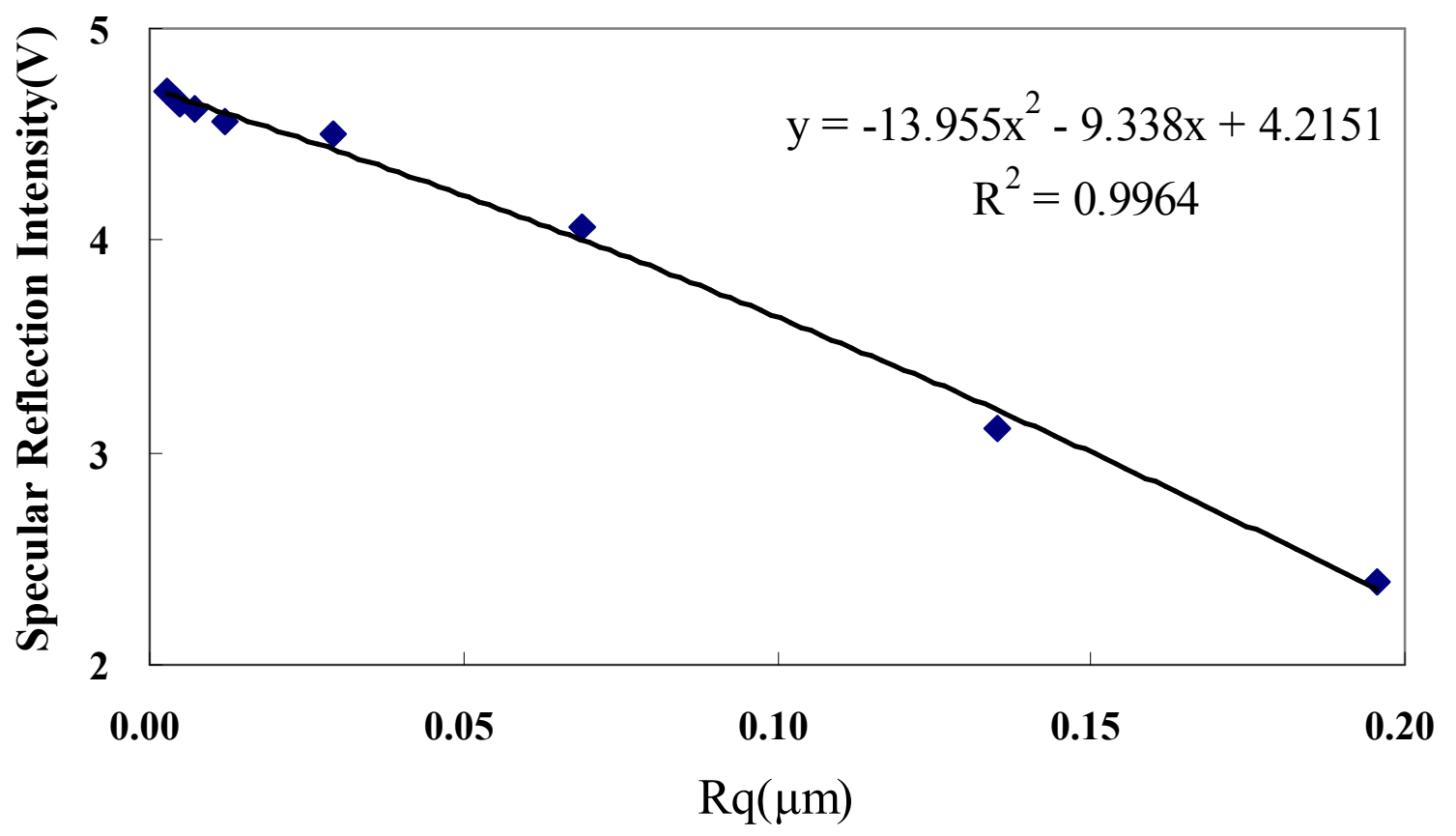

Fig. 4 The specular reflection intensity from the machined electroless nickel at an incident Angle of $45-{ }^{\circ}$ and $26-\mathrm{mm}$ Distance by green laser of $532 \mathrm{~nm}$.

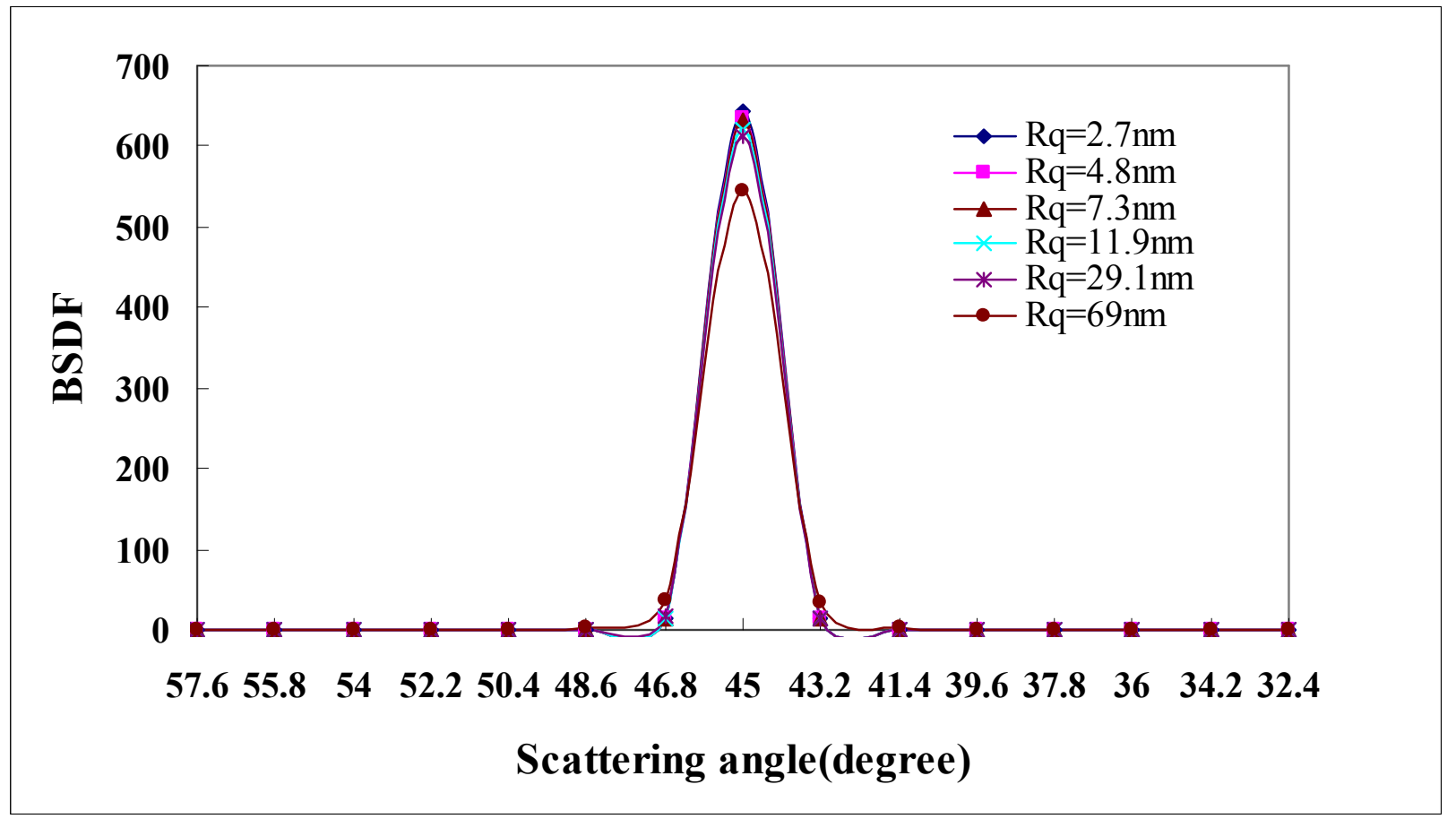

Fig. 5 (a) Light Scattering Reflectance from the Machined Electroless nickel at an Incident Angle of 45- ${ }^{\circ}$ and 26-mm Distance 


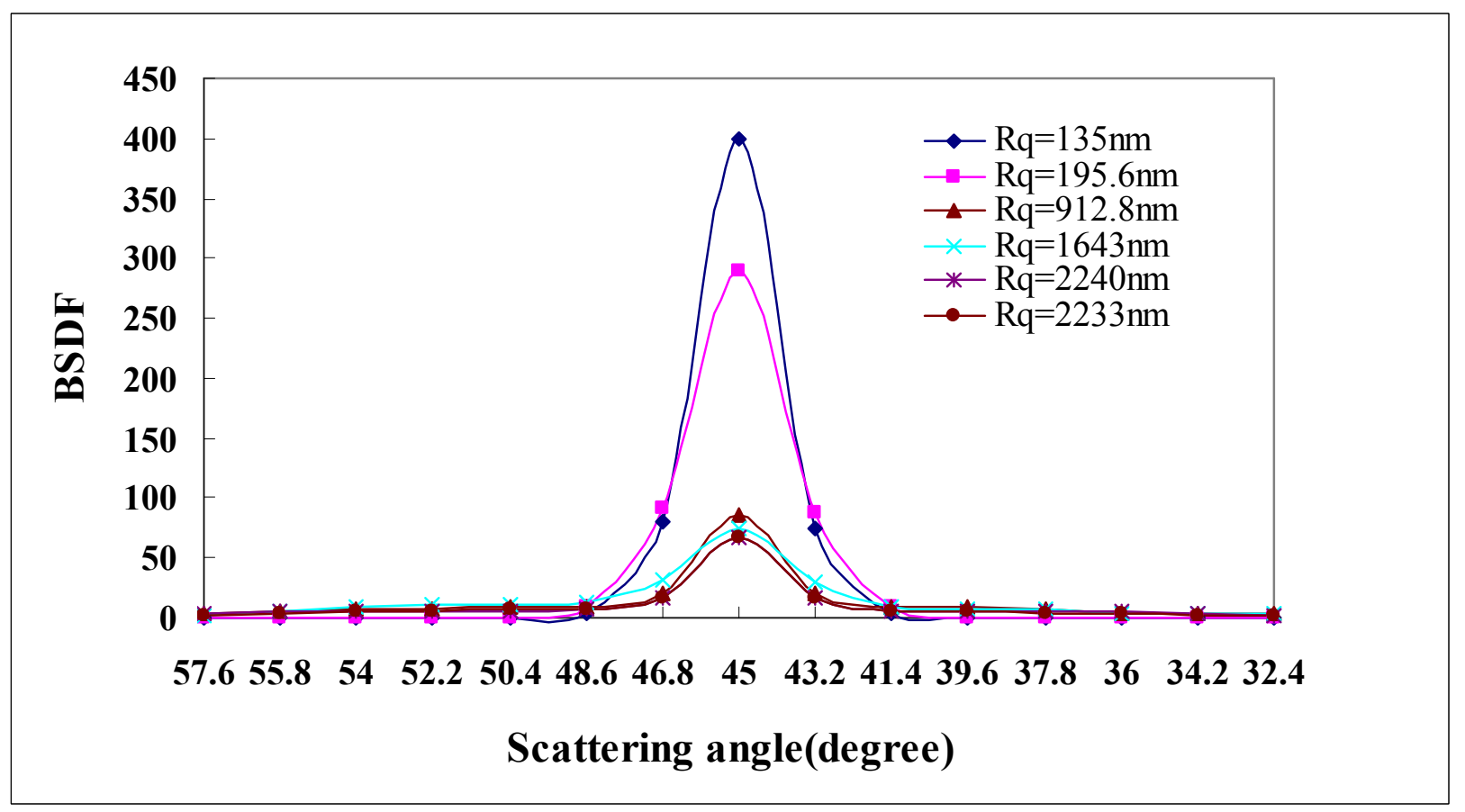

Fig. 5 (b) Light Scattering Reflectance from the Machined Electroless nickel at an Incident Angle of 45- ${ }^{\circ}$ and 26-mm Distance

\section{Conclusions}

The received intensity of the specular channel was found to increase with decrease in the surface roughness, which is in agreement with earlier studies. In addition, the scattering light was found to be of Gaussian distribution once the surface roughness became smaller. This phenomenon of the light scattering distribution became wider when the surface roughness was becoming worse. However, a larger distance was found to enhance the BSDF values owing to a larger receiving angle of each element of the photodiode array, i.e., for each element of the photodiode, the receiving angle increased with respect to the increased distance. The BSDF of the machined surface was found to be proportional to the roughness. Using the ratio of the main and side measuring channels of the photodiode array, a suitable approach to determine the relationship between light scattering and surface roughness has been developed. Thus, the laser and the photodiode array effectively predicted the roughness of the ultraprecision machined surfaces of the electroless nickel. This study developed a feasible online measurement technique to determine the roughness of ultraprecision machined surface by reflected light scattering effect.

\section{Acknowledgments}

Special thanks would be brought to the National Science Council of 97-2221-E-131-011 for the financial support.

\section{References}

1. Beckmann, P and Spizzichino, A., 1987, The scattering of electromagnetic waves from rough surfaces Norwood, MA: Artech House, inc

2. Bennett, J. M., 1992, Surface finishand its measurement, Part A and B. Washington, DC: Optical Society of America 
3. Bennett, J. M. and Mattson, L., 1989. Introduction to Surface Roughness and Scattering. Optical Society of America

4. Cahill, B. and Baradie, M. A. El, 2001. LED-based fibre-optic sensor for measurement of surface roughness. J. of Materials Processing Technology, Vol.119, No.1-3, pp. 299-306

5. Elson, J. M. and Bennett, J. M. "Relation between the angular dependence of scattering and the statistical properties of optical surfaces," J. Opt. Soc. Am. 69, 31-47 (1979)

6. Fontani D. et al., 1999. Optical Control of Surface Finish. Optics and Lasers in Engineering, Vol.32, No. 5, pp. 459-472

7. Fontani, D. et al., 2001. Control of crankshaft finish by scattering technique. Optics and Lasers in Engineering, Vol. 35, Issue 6, pp.387-396

8. Luk,F., Huynh, V. and North., W., 1989. Measurement of surface roughness by a machine vision system. J. of Physics E-Scientific Instruments, Vol. 22, pp.977 980

9. Persson, U.,1998. In-Process Measurement of Surface Roughness using Light Scattering. Wear, Vol.215

10. Rao, C. B. and Raj, B., 2003. Study of engineering surfaces using laser-scattering techniques. Sadhana, Vol.28, pp.739-761

11. Stover, J. C., 1995, Optical Scattering: Measurement and Analysis-2nd edition, SPIE Press, pp. $19-22$

12. Wang, S. H., Quan, C., Tay, C. J. and Shang, H. M., 2000. Surface roughness measurement in the submicrometer range using laser scattering. Opt Eng, Vol.39, pp.1597 1601

13. Tanner, L. H. and Fahoum, M. A., 1976. A study of the surface parameters of ground and lapped metal surface using specular and diffuse reflection of laser light. Wear, Vol.36, pp.299316

14. Tay, C. J., Wang, S. H. et al., 2004. Surface roughness investigation of semi-conductor wafers. Opt. Laser Technol, Vol.36, pp. 535-539

15. Vandembroucq, D. et al., 2001. Light scattering from cold rolled aluminum surfaces. Optics Communications, Vol.187, pp.289-294

16. Vorburger, T.V., 1987. Measurement of Roughness of very Smooth Surfaces. CIRP Ann., Vol.36,pp.503 509

17. Zhao, X. Z. and Gao, Z., 2009. Surface roughness measurement using spatial-average analysis of objective speckle pattern in specular direction. Optics and Lasers in Engineering, Vol. 47, No.11, pp.1307-1316 